\title{
A First-Order $\varepsilon$-Approximation Algorithm for Linear Programs and a Second-Order Implementation
}

\author{
Ana Maria A.C. Rocha ${ }^{1}$, Edite M.G.P. Fernandes ${ }^{1}$, and João L.C. Soares ${ }^{2}$ \\ 1 Departamento de Produção e Sistemas, Universidade do Minho, Portugal, \\ \{arocha; emgpf\}@dps . uminho.pt \\ 2 Departamento de Matemática, Universidade de Coimbra, Portugal, \\ jsoares@mat.uc.pt
}

\begin{abstract}
This article presents an algorithm that finds an $\varepsilon$-feasible solution relatively to some constraints of a linear program. The algorithm is a first-order feasible directions method with constant stepsize that attempts to find the minimizer of an exponential penalty function. When embedded with bisection search, the algorithm allows for the approximated solution of linear programs. We present applications of this framework to set-partitioning problems and report some computational results with first-order and second-order implementations.
\end{abstract}

\section{Introduction}

Set-covering, -partitioning and -packing models arise in many applications like crew scheduling (trains, buses or airplanes), political districting, protection of microdata, information retrieval, etc. Typically these models are suboptimally solved by heuristics because an optimization framework (usually of the branchand-price type) has to be rather specialized, if feasible at all. Moreover, a branchand-price framework requires the solution of large linear programs at every node of the branch-and-price tree and these linear programs may take a long time and storage to be solved to optimality.

Our framework attempts to find reasonable approximate solutions to those models quickly and without too much storage, along the lines of Lagrangian relaxation. The approximation obtained may serve the purpose of speeding-up the optimal basis identification by simplex-type algorithms. We will be looking for an approximated solution of a linear program in the following form

$$
\begin{aligned}
z_{*} \equiv \min & c x \\
\text { s.t. } & A x \geq b, \\
& x \in P,
\end{aligned}
$$

where $A$ is a real $m \times n$ matrix, $b$ is a real $m$-dimensional vector and $P \subseteq \mathbb{R}^{n}$ is a compact set (possibly, a lattice) over which optimizing linear programs is considered "easy". For example, in a set-covering model, $A$ is a matrix of zeros 
and ones, $b$ is a vector of all-ones, and the set $P$ is the lattice $\{0,1\}^{n}$, or the hypercube $[0,1]^{n}$ in the fractional version. If $P$ includes a budget constraint then $P$ becomes the feasible region of a $0-1$ knapsack problem or a fractional $0-1$ knapsack problem, respectively. We also assume that $\operatorname{conv}(P)$ is a polyhedron.

In recent years, several researchers have developed algorithms (see $[3,4,2]$ ) that seek to produce approximate solutions to linear programs of this sort, by constructing an exponential potential function which serves as a surrogate for feasibility and optimality.

We focus on obtaining a reasonable approximation to the optimal solution of (1) by an $\varepsilon$-feasible solution. We say that a point $x$ is $\varepsilon$-feasible relatively to the constraints $A x \geq b$ if

$$
\lambda(x) \equiv \max _{i=1, \ldots, m}\left(b_{i}-a_{i} x\right) \leq \varepsilon
$$

where $a_{i}$ denotes the $i$ th row of the matrix $A$. To achieve this, we will attempt to solve the following convex program

$$
\begin{aligned}
\Phi(\alpha, z)= & \min \phi_{\alpha}(x) \equiv \sum_{i=1}^{m} \exp \left(\alpha\left(b_{i}-a_{i} x\right)\right) \\
& \text { s.t. } x \in P^{\prime}(z) \equiv \operatorname{conv}(P \cap\{x: c x \leq z\})
\end{aligned}
$$

where $\operatorname{conv}(\cdot)$ denotes convex hull, for several adequate values of the parameters $\alpha$ and $z$. The scalar $\alpha$ is a penalty parameter and $z$ is a guess for the value of $z_{*}$. To solve the nonlinear program (3) we propose a first-order feasible directions method with constant stepsize whose running time depends polynomially on $1 / \varepsilon$ and the width of the set $P^{\prime}(z)$ relatively to the constraints $A x \geq b$. Significantly, the running time does not depend explicitly on $n$ and, hence, it can be applied when $n$ is exponentially large, assuming that, for a given row vector $y$, there exists a polynomial subroutine to optimize $y A x$ over $P^{\prime}(z)$.

This paper is organized as follows. In Sect. 2 we describe the $\varepsilon$-approximation algorithm used in this work. Then, in Sect. 3 we present the first-order algorithm with fixed stepsize. Our computational experience on set-partitioning problems is presented in Sect. 4 and Sect. 5 contains some conclusions and future work.

\section{Main $\varepsilon$-Approximation Algorithm}

If $x$ is feasible in (1) then $\phi_{\alpha}(x) \leq m$, while if $x$ is simply $\varepsilon$-feasible then $\phi_{\alpha}(x) \leq$ $m \exp (\alpha \varepsilon)$. On the other hand, if $x$ is not $\varepsilon$-feasible then $\phi_{\alpha}(x)>\exp (\alpha \varepsilon)$. We will choose $\alpha$ so that it may be possible to assert whether $x$ is $\varepsilon$-feasible from the value of $\phi_{\alpha}(x)$, as formally stated in the next lemma.

Lemma 1. If $\alpha \geq \ln ((1+\varepsilon) m) / \varepsilon$ then,

1. if there is no $\varepsilon$-feasible solution relatively to 'Ax $\geq b^{\prime}$ 'such that $x \in P^{\prime}(z)$ then $\Phi(\alpha, z)>(1+\varepsilon) m$.

2. if $\phi_{\alpha}(x) \leq(1+\varepsilon) m$ then $x$ is an $\varepsilon$-feasible solution relatively to ' $A x \geq b$ '. 
Proof. If there is no $\varepsilon$-feasible solution relatively to ' $A x \geq b$ ' such that $x \in P^{\prime}(z)$ then $\varepsilon<\varepsilon^{\prime} \equiv \min \left\{\lambda(x): x \in P^{\prime}(z)\right\}$. Thus, $\Phi(\alpha, z)>\exp \left(\alpha \varepsilon^{\prime}\right) \geq(1+\varepsilon) m$. If $\phi_{\alpha}(x) \leq(1+\varepsilon) m$ then $\exp \left(\alpha\left(b_{i}-a_{i} \bar{x}\right)\right) \leq(1+\varepsilon) m$, for any $i=1, \ldots, m$. Equivalently, $b_{i}-a_{i} \bar{x} \leq \ln ((1+\varepsilon) m) / \alpha \leq \varepsilon$, for any $i=1, \ldots, m$.

Keeping the value of $\alpha$ fixed, we will use bisection to search for the minimum value of $z$ such that $P^{\prime}(z) \cap\{x: A x \geq b\}$ is nonempty, being driven by (3). The bisection search maintains an interval $\left[z_{a}, z_{b}\right]$ such that $P^{\prime}\left(z_{a}\right) \cap\{x: A x \geq b\}$ is empty, and there is some $x \in P^{\prime}\left(z_{b}\right)$ that is $\varepsilon$-feasible relatively to ' $A x \geq b$ '. The search is interrupted when $z_{b}-z_{a}$ is small enough to guarantee $z_{b} \leq z_{*}$. This does not imply any bound on how much $z_{b}$ differs from $z_{*}$. It may be possible that $z_{b}$ is much less than $z_{*}$ though very unlikely for $\alpha$ large, as it is implicit from Proposition 1 below.

Proposition 1. Let the sequence $\left\{\alpha_{k}\right\}$ be such that $\lim _{k} \alpha_{k}=+\infty$ and $y^{k}$ be a vector defined componentwise by

$$
y_{i}^{k}=\alpha_{k} \exp \left(\alpha_{k}\left(b_{i}-a_{i} x^{k}\right)\right) \quad(i=1,2, \ldots, m),
$$

where $x^{k}$ is optimal in $\Phi\left(\alpha_{k}, z_{*}\right)$. Then, for every accumulation point $(\bar{x}, \bar{y})$ of the sequence $\left\{\left(x^{k}, y^{k}\right)\right\}, \bar{x}$ is optimal for program (1).

Proof. Assume $\lim _{k \in K}\left(x^{k}, y^{k}\right)=(\bar{x}, \bar{y})$. Since $P$ is closed, $\bar{x} \in P^{\prime}\left(z_{*}\right)$. If we show that $A \bar{x} \geq b$ then $\bar{x}$ must be optimal in (1). Since $\lim _{k \in K} \alpha_{k} \exp \left(\alpha_{k}\left(b_{i}-a_{i} x^{k}\right)\right)$ exists then $\bar{y}_{i}=\lim _{k \in K} \alpha_{k} \exp \left(\alpha_{k}\left(b_{i}-a_{i} \bar{x}\right)\right)$, from where we conclude that $a_{i} \bar{x} \leq b_{i}$, for every $i=1,2, \ldots, m$.

If $\left[z_{a}^{k}, z_{b}^{k}\right]$ is the last bisection interval of the search for a given $\varepsilon_{k}$ then, we may restart the bisection search with $\left[z_{b}^{k}, z_{b}\right]$ for some $\varepsilon_{k+1}<\varepsilon_{k}$, for example, $\varepsilon_{k+1}=\varepsilon_{k} / 2$. The value of $z_{b}$ denotes a proven upper bound on the value of $z_{*}$. Note that an $\varepsilon_{k}$-feasible solution is known at the left extreme of the new interval and furthermore it belongs to $P^{\prime}(z)$, for any $z \in\left[z_{b}^{k}, z_{b}\right]$. Thus, it may serve as initial solution for the minimization of $\phi_{\alpha}$ when $\varepsilon=\varepsilon_{k+1}$ and will not overflow the exponential function evaluations.

Each iteration of our main algorithm consists of a number of iterations of bisection search on the $z$ value for a fixed value of the parameter $\varepsilon$. Then, $\varepsilon$ is decreased, the bisection interval is updated and bisection search restarts. This process is terminated when $\varepsilon$ is small enough.

From Lemma 1, if $\Phi(\varepsilon, z) \leq(1+\varepsilon) m$ then there is $x \in P^{\prime}(z)$ that is $\varepsilon$-feasible relatively to ' $A x \geq b$ ' (for example, the optimal solution); otherwise, there is no feasible solution $x$ in (1) satisfying $c x \leq z$. The new interval $\left[z_{a}^{k+1}, z_{b}^{k+1}\right]$ is adequately updated and $k$ is increased by one unit. Termination occurs when $z_{b}^{k}-z_{a}^{k}$ is small enough. The algorithm is formally described in Algorithm 1. Step 2 of the algorithm Bisection search involves applying an off-the-shelf convex optimization method to (3). Note that, we always have $x_{a}^{k} \in P^{\prime}(z)$ which makes it a natural starting point for the corresponding optimization algorithm. 
Algorithm 1. Bisection search $\left(\varepsilon, x_{a}, z_{a}, x_{b}, z_{b}\right)$

Given $0<\varepsilon$, and $x_{a} \in P^{\prime}\left(z_{a}\right), x_{b} \in P^{\prime}\left(z_{b}\right)$ such that

$$
\Phi\left(\varepsilon, z_{b}\right) \leq \phi_{\varepsilon}\left(x_{b}\right) \leq(1+\varepsilon) m<\Phi\left(\varepsilon, z_{a}\right) \leq \phi_{\varepsilon}\left(x_{a}\right) .
$$

Initialization: $\quad$ Set $\left(x_{a}^{0}, z_{a}^{0}, x_{b}^{0}, z_{b}^{0}\right):=\left(x_{a}, z_{a}, x_{b}, z_{b}\right)$ and $k:=0$.

Generic Iteration $k$ :

Step 1: If $z_{b}^{k}-z_{a}^{k}=1$ then set $\left(x_{a}, z_{a}, x_{b}, z_{b}\right):=\left(x_{a}^{k}, z_{a}^{k}, x_{b}^{k}, z_{b}^{k}\right)$ and STOP.

Step 2: Set $z:=\left\lceil\left(z_{a}^{k}+z_{b}^{k}\right) / 2\right\rceil$ and obtain $\bar{x} \in P^{\prime}(z)$ such that

$$
\begin{aligned}
\text { either } & \Phi(\varepsilon, z) \leq \phi_{\varepsilon}(\bar{x}) \leq(1+\varepsilon) m, \\
\text { or } \quad & (1+\varepsilon) m<\Phi(\varepsilon, z) \leq \phi_{\varepsilon}(\bar{x}) .
\end{aligned}
$$

Step 3: Set $\left(x_{a}^{k+1}, z_{a}^{k+1}, x_{b}^{k+1}, z_{b}^{k+1}\right):=\left\{\begin{array}{l}\left(x_{a}^{k}, z_{a}^{k}, \bar{x}, z\right) \text { if (6) holds, } \\ \left(\bar{x}, z, x_{b}^{k}, z_{b}^{k}\right) \text { if (7) holds. }\end{array}\right.$

Set $k:=k+1$.

We may now present a formal description of the Main algorithm (see Algorithm 2). On entry: $z_{a}=\lceil\min \{c x-y(A x-b): x \in P\}\rceil$ is an integral lower bound on the value of $z_{*}$, for some fixed $y \geq 0 ; x_{a} \in P^{\prime}\left(z_{a}\right)$ and $\Delta$ is a positive integer related to the initial amplitude of the starting intervals in each bisection search. Overall, we have the following convergence result. After a finite number of calls, the last interval $\left[z_{b}-1, z_{b}\right]$ of the Bisection search routine is such that $z_{b}=\lceil\bar{z}\rceil$, where $\bar{z}=\min \left\{z: x \in P^{\prime}(z), A x \geq b\right\}$. In general $\bar{z} \leq z_{*}$, with equality if $P$ is a polyhedron.

Algorithm 2. Main $\left(z_{a}, x_{a}, z_{b}, x_{b}, \Delta\right)$

Given $z_{a} \leq z_{*}, x_{a} \in P^{\prime}\left(z_{a}\right)$ and a positive integer $\Delta$.

Initialization:

Choose $\varepsilon>0$ so that $x_{a}$ do not overflow $\phi_{\varepsilon}\left(x_{a}\right)$ and $\Phi\left(\varepsilon, z_{a}\right)>(1+\varepsilon) m$.

While $\Phi\left(\varepsilon, z_{a}+\Delta\right)>(1+\varepsilon) m$ redefine $x_{a}$ and set $z_{a}:=z_{a}+\Delta$.

Define $x_{b}$ as the last solution found and set $z_{b}:=z_{a}+\Delta$.

Generic Iteration:

Step 1: Call Bisection search $\left(\varepsilon, x_{a}, z_{a}, x_{b}, z_{b}\right)$.

Step 2: While $\left(\Phi\left(\varepsilon / 2, z_{b}\right) \leq(1+\varepsilon / 2) m\right.$ and $\varepsilon$ is not small enough) redefine $x_{b}$ and set $\varepsilon:=\varepsilon / 2$.

If ( $\varepsilon$ is small enough)

Then set $x_{b}$ as the last solution found and STOP.

Step 3: Set $x_{a}:=x_{b}$ and $z_{a}:=z_{b}$.

While $\left(\Phi\left(\varepsilon, z_{b}+\Delta\right)>(1+\varepsilon) m\right)$ redefine $x_{a}, z_{a}$ and set $z_{b}:=z_{b}+\Delta$.

Set $x_{b}$ as the last solution found and set $z_{b}:=z_{b}+\Delta$.

Repeat Step 1.

\section{A First-Order Algorithm with Fixed Stepsize}

Our conceptual algorithm to solving (3) is a first-order iterative procedure with a fixed stepsize. The algorithm coincides with the algorithm Improve-Packing 
proposed in [5] but the stepsize and the stopping criterion are different. The direction of movement at a generic iterate $\bar{x} \in P^{\prime}(z)$, that is not $\varepsilon$-feasible relatively to the constraints ' $A x \geq b$ ', is determined from solving the following linear program

$$
\begin{aligned}
& \min \phi_{\varepsilon}(\bar{x})+\nabla \phi_{\varepsilon}(\bar{x})(x-\bar{x}) \\
& \text { s.t. } x \in P^{\prime}(z) .
\end{aligned}
$$

If $\hat{x}$ is optimal in (8) then we reset $\bar{x}$ to $\bar{x}+\hat{\sigma}(\hat{x}-\bar{x})$, for some fixed stepsize $\hat{\sigma} \in$ $(0,1]$, and proceed analogously to the next iteration. The conceptual algorithm is halted when $\phi_{\varepsilon}(\bar{x}) \leq(1+\varepsilon) m$ or a maximum number of iterations is reached. Of course, in practice other stopping criteria should be accounted for. For example, notice that (8) is equivalent to

$$
\begin{aligned}
& \max (\bar{y} A) x \\
& \text { s.t. } \quad c x \leq z, \\
& \\
& x \in P,
\end{aligned}
$$

for $\bar{y}$ defined componentwise by $\bar{y}_{i}=\alpha \exp \left(\alpha\left(b_{i}-a_{i} \bar{x}\right)\right)$, for $i=1,2, \ldots, m$. This is essentially because $\nabla \phi_{\varepsilon}(\bar{x})=-\bar{y} A$. Then, since $\bar{y} \geq 0$, the inequality ' $\bar{y} A x \geq \bar{y} b$ ' is valid for the polyhedron $\{x: A x \geq b\}$. Thus, if at some point of the solution procedure of (3) we have that the optimal value of (9) is smaller than $\bar{y} b$ then we may immediately conclude that $P^{\prime}(z) \cap\{x: A x \geq b\}$ is empty.

Theorem 1 below presents one particular choice for the fixed stepsize $\hat{\sigma}$. It depends on the following quantity, introduced as the width of $P^{\prime}(z)$ relatively to the constraints ' $A x \geq b$ ' in [5],

$$
\rho \equiv\left\{\begin{array}{c}
\sup \|A x-A y\|_{\infty} \\
\text { s.t. } x, y \in P^{\prime}(z)
\end{array}\right\}=\left\{\max _{i=1,2, \ldots, m}\left(\begin{array}{c}
\sup \left|a_{i} x-a_{i} y\right| \\
\text { s.t. } x, y \in P^{\prime}(z)
\end{array}\right)\right\} .
$$

In [5], $\rho$ is defined differently depending when whether the matrix $A$ is such that $A x \geq 0$, for every $x \in P^{\prime}(z)$, or not. If yes, then the two definitions coincide with $\rho=\max _{i} \max _{x \in P^{\prime}(z)} a_{i} x$. If not, then our definition of $\rho$ is half of the $\rho$ that is proposed in [5].

Theorem 1. ([6]) Assume that $z_{*} \leq z, \bar{x} \in P^{\prime}(z)$ and it is not $\varepsilon$-feasible (relatively to ' $\left.A x \geq b^{\prime}\right)$, for some $\varepsilon \in(0,1), \hat{x} \in P^{\prime}(z)$ is optimal for program (8), $\rho$ is given by (10) and

$$
\alpha \geq \max \left(\frac{\ln (m(3+\varepsilon))}{\varepsilon}, \frac{1}{\rho \ln 2}\right) .
$$

Then, for $\hat{\sigma}=1 /(\alpha \rho)^{2}$ we have that

$$
\phi_{\varepsilon}(\bar{x}+\hat{\sigma}(\hat{x}-\bar{x}))<\left(1-\frac{1}{4(\alpha \rho)^{2}} \frac{1+\varepsilon}{3+\varepsilon}\right) \phi_{\varepsilon}(\bar{x}) .
$$

In summary, assuming that $z_{*} \leq z$, if the first $k$ iterates are not $\varepsilon$-feasible then

$$
\phi_{\varepsilon}\left(x^{k+1}\right)<\left(1-\frac{1}{4(\alpha \rho)^{2}} \frac{1+\varepsilon}{3+\varepsilon}\right)^{k} \phi_{\varepsilon}\left(x^{0}\right)
$$


where, we note, that the right hand side goes to zero as $k$ goes to $+\infty$. The following corollary states a worst-case complexity bound on the number of iterations of the algorithm.

Corollary 1. ([6]) If $\alpha$ satisfies (11) and $\varepsilon \in(0,1)$ then our algorithm, using $\hat{\sigma}=1 /(\alpha \rho)^{2}$ and starting from $x^{0} \in P^{\prime}(z)$, terminates after

$$
\frac{\ln (m)+\ln (1+\varepsilon)-\ln \phi_{\varepsilon}\left(x^{0}\right)}{\ln \left(1-\frac{1}{4(\alpha \rho)^{2}} \frac{1+\varepsilon}{3+\varepsilon}\right)}<16 \alpha^{3} \rho^{2} \lambda\left(x^{0}\right)
$$

iterations, with an $x \in P^{\prime}(z)$ that is $\varepsilon$-feasible relatively to 'A $A \geq b^{\prime}$ ' or, otherwise, with the proof that there is no $x$ feasible in (1) such that $c x \leq z$.

Note that the right hand side of (14) is $\mathcal{O}\left(\ln ^{3}(m) \varepsilon^{-3} \rho^{2} \lambda\left(x^{0}\right)\right)$. If $\lambda\left(x^{0}\right)=\mathcal{O}(\varepsilon)$ then only $\mathcal{O}\left(\ln ^{3}(m) \varepsilon^{-2} \rho^{2}\right)=\tilde{\mathcal{O}}\left(\varepsilon^{-2} \rho^{2}\right)$ iterations are required. This complexity result is related to Karger and Plotkin's [4, Theorem 2.5] $\left(\tilde{\mathcal{O}}\left(\varepsilon^{-3} \rho\right)\right)$ and Plotkin, Shmoys and Tardos's [5, Theorem 2.12] $\left(\tilde{\mathcal{O}}\left(\varepsilon^{-2} \rho \ln \varepsilon^{-1}\right)\right)$. The result of Karger and Plotkin is valid even if the budget constraint is included in the objective function of (3) without counting in the definition of $\rho$. We recall that a function $f(n)$ is said to be $\tilde{\mathcal{O}}(g(n))$ if there exists a constant $c$ such that $f(n) \ln ^{c}(n) \geq$ $\mathcal{O}(g(n))$.

Given an initial point $\bar{x} \in P^{\prime}(z)$, the Algorithm 3 contains a practical implementation of the first-order algorithm to solving (3).

Algorithm 3. Solve_subproblem ( $\bar{x}$, flag)

Given $\bar{x} \in P^{\prime}(z)$ and a small positive tolerance $\delta$.

Generic Iteration:

Step 1: If $\left(\phi_{\varepsilon}(\bar{x}) \leq(1+\varepsilon) m\right)$

Then set flag $:=\mathbf{C P 1}$ and STOP;

Else If (maximum number of iterations is reached)

Then set flag $:=\mathbf{C P} 4$ and STOP.

Step 2: Let $\hat{x}$ be optimal for (9).

If $\left(\phi_{\varepsilon}(\bar{x})+\nabla \phi_{\varepsilon}(\bar{x})(\hat{x}-\bar{x})>(1+\varepsilon) m\right)$

Then set flag $:=\mathbf{C P 2}$ and STOP;

Else If $\left.\left(\phi_{\varepsilon}(\bar{x})+\nabla \phi_{\varepsilon}(\bar{x})(\hat{x}-\bar{x})>\Phi_{\varepsilon}(\bar{x})-\delta\right)\right)$

Then set flag $:=$ CP3 and STOP;

Else If $(\bar{y} A \hat{x}<\bar{y} b)$

Then set flag $:=\mathbf{C P 5}$ and STOP.

Step 3: Set $\bar{x}:=\bar{x}+\sigma_{*}(\hat{x}-\bar{x})$,

where $\sigma_{*} \in \arg \min \left\{\phi_{\varepsilon}(\bar{x}+\sigma(\hat{x}-\bar{x})): \sigma \in(0,1]\right\}$.

Repeat Step 1.

On exit of this routine, $\bar{x}$ should be understood as optimal in (3) when flag $\neq \mathbf{C P} 4$. When this is the case, output should be interpreted as follows: $\Phi(\varepsilon, z) \leq(1+\varepsilon) m \Longleftrightarrow$ flag $\in\{\mathbf{C P 1}, \mathbf{C P} 3\}$.

Step 2 of the routine Solve_subproblem requires solving a program with a linear objective function. Interesting possibilities are: (1) $P=[0,1]^{n}$, in which 
case (9) can be solved by a greedy type algorithm; (2) $P=\{0,1\}^{n}$, in which case (9) is a 0-1 knapsack problem and can be solved by the CPLEX MIP solver; (3) $P$ is a generic polyhedron in which case (9) can be solved by the CPLEX solver.

In what follows we will expand on how the line search is performed assuming that we are looking for an $\varepsilon$-feasible solution relatively to an equality system $A x=b$, as this is the case with the test problems studied. Step 3 of the algorithm Solve_subproblem aims at finding a minimizer $\sigma_{*}$ of the function $g:[0,1] \rightarrow \mathbb{R}$ defined by $g(\sigma) \equiv \phi_{\varepsilon}(\bar{x}+\sigma d) \equiv \sum_{i=1}^{m} \phi_{i}(\bar{x}+\sigma d) \equiv 2 \sum_{i=1}^{m} \cosh \left(a_{i} \bar{x}-b_{i}+\right.$ $\left.\sigma a_{i} d\right)$, where $d=\hat{x}-\bar{x}$ and cosh denotes de hyperbolic cosine function. Note that $g$ is convex and $g^{\prime}(0)<0$. The numerical method of choice would be the unidimensional Newton's method for it is, in this case, globally convergent and possesses locally quadratic convergence.

However, since the functions $\phi_{i}$ are defined by exponential functions, Newton's method may require too many iterations to reach its region of quadratic convergence. A method like bisection search may be more adequate at early stages of the minimization process. Our initial bisection interval is implied by the following result.

Theorem 2. ([6]) For every $i \in\{1,2, \ldots, m\}$, let $U_{i}$ be an upper bound on the value of $\phi_{i}\left(\bar{x}+\sigma_{*} d\right)$. Then, for every $i$ such that $a_{i} d \neq 0$, the following holds,

$$
\sigma_{*} \leq \frac{1}{\alpha a_{i} d} \ln \left(\frac{U_{i}+\operatorname{sgn}\left(a_{i} d\right) \sqrt{U_{i}^{2}-4}}{2 \exp \left(\alpha\left(a_{i} \bar{x}-b_{i}\right)\right)}\right) .
$$

where $\operatorname{sgn}(\cdot)$ denotes the sign function.

The bound (15) is important for the search for $\sigma_{*}$ not to overflow the exponential function evaluations.

Bienstock [2] proposed to use bisection search until a sufficiently small interval is found and only then start with Newton's method. We propose a slightly different procedure. We propose to try two Newton's steps departing from the left limit of the current bisection interval. By using these two Newton points we make a judgement of whether the region of quadratic convergence for Newton's method was achieved. If yes, we switch to the pure Newton's method. More precisely, if $\sigma_{\mathrm{N} 1}$ is the first Newton point and $\sigma_{\mathrm{N} 2}$ is the second Newton point then we consider that the region of quadratic convergence is achieved if

$$
\left(c_{1} g^{\prime}\left(\sigma_{\mathrm{N} 2}\right)^{2} \leq\left|g^{\prime}\left(\sigma_{\mathrm{N} 1}\right)\right| \text { if }\left|g^{\prime}\left(\sigma_{\mathrm{N} 2}\right)\right|>1\right) \text { or }\left(\left|g^{\prime}\left(\sigma_{\mathrm{N} 2}\right)\right| \leq c_{2} g^{\prime}\left(\sigma_{\mathrm{N} 1}\right)^{2} \text { if }\left|g^{\prime}\left(\sigma_{\mathrm{N} 2}\right)\right| \leq 1\right)
$$

where $c_{1}, c_{2}$ are positive constants. Otherwise, the interval is updated by using the information on these points, the midpoint $\sigma_{\text {mid }}$ is also tried and the bisection interval is again updated. The process restarts.

\section{Computational Results with Set-Partitioning Problems}

In this section we report computational results for the first-order method previously discussed and with a second-order nonlinear programming package. The 
computational tests were performed on a PC with a $2.66 \mathrm{GHz}$ Pentium IV microprocessor and $512 \mathrm{Mb}$ of memory running RedHat Linux 8.0. The algorithm was implemented in AMPL (Version 7.1) modeling language.

Here, we are looking for finding "good" $\varepsilon$-feasible solutions of fractional setpartitioning problems arising in airline crew scheduling. Some of these linear programs are extremely difficult to solve with traditional algorithms. Generically, fractional set-partitioning problems are of the form

$$
\begin{aligned}
& \min c x \\
& \text { s.t. } A x=\mathbb{1} \\
& \\
& x \in[0,1]^{n},
\end{aligned}
$$

where $A$ is a $m \times n$ matrix, with $0-1$ coefficients, and $\mathbb{1}$ denotes a vector of ones. Our framework was tested on real-world set-partitioning problems obtained from the OR-Library (http://www.brunel.ac.uk/depts/ma/research/jeb/info.html).

Before a call to Main algorithm we apply the volume algorithm, developed by Barahona and Anbil [1], that is an extension of the subgradient algorithm, which produces approximate feasible primal and dual solutions to a linear program, much more quickly than solving it exactly. This algorithm approximately solves the Lagrangian relaxation of the problem (17), i.e.,

$$
\begin{aligned}
& \max \min \left\{c x-y(A x-\mathbb{1}): x \in[0,1]^{n}\right\} \\
& \text { s.t. } y \in \mathbb{R}^{m}
\end{aligned}
$$

requiring a not too demanding number of iterations.

The main characteristics of the selected problems are described in Table 1.

Table 1. Set-partitioning problems

\begin{tabular}{lccrcrrrr}
\hline Name & $m$ & $n$ & $z^{\text {LP }}$ & $\begin{array}{c}\text { Vol } \\
\text { It. }\end{array}$ & $\begin{array}{c}\text { Vol } \\
\text { Dual }\end{array}$ & $\begin{array}{c}\text { Vol } \\
\text { Primal }\end{array}$ & $\begin{array}{c}\text { Vol } \\
\text { Viol. }\end{array}$ & $\begin{array}{c}\text { Vol } \\
\text { Time }\end{array}$ \\
\hline sppnw08 & 24 & 434 & 35894 & 356 & 35894.0 & 36188.0 & 0.01889 & 0.02 \\
sppnw10 & 24 & 853 & 68271 & 501 & 68146.8 & 68510.8 & 0.01974 & 0.04 \\
sppnw12 & 27 & 626 & 14118 & 447 & 14101.4 & 14222.6 & 0.01859 & 0.03 \\
sppnw15 & 31 & 467 & 67743 & 483 & 67713.8 & 67407.0 & 0.01934 & 0.03 \\
sppnw20 & 22 & 685 & 16626 & 408 & 16603.4 & 16645.2 & 0.01991 & 0.02 \\
sppnw21 & 25 & 577 & 7380 & 358 & 7370.8 & 7387.1 & 0.01875 & 0.03 \\
sppnw22 & 23 & 619 & 6942 & 340 & 6916.8 & 6917.4 & 0.01903 & 0.02 \\
sppnw23 & 19 & 711 & 12317 & 357 & 12300.1 & 12412.8 & 0.01971 & 0.03 \\
sppnw24 & 19 & 1366 & 5843 & 313 & 5827.8 & 5885.3 & 0.01924 & 0.04 \\
sppnw25 & 20 & 1217 & 5852 & 305 & 5829.8 & 5851.3 & 0.01763 & 0.04 \\
sppnw26 & 23 & 771 & 6743 & 304 & 6732.8 & 6742.4 & 0.01584 & 0.03 \\
sppnw27 & 22 & 1355 & 9877.5 & 363 & 9870.0 & 9934.8 & 0.01933 & 0.05 \\
sppnw28 & 18 & 1210 & 8169 & 342 & 8160.1 & 8167.5 & 0.01995 & 0.04 \\
sppnw32 & 19 & 294 & 14570 & 430 & 14559.9 & 14559.6 & 0.01748 & 0.01 \\
sppnw35 & 23 & 1709 & 7206 & 334 & 7194.0 & 7262.6 & 0.01633 & 0.06 \\
sppnw36 & 20 & 1783 & 7260 & 631 & 7259.2 & 7225.3 & 0.01995 & 0.13 \\
sppnw38 & 23 & 1220 & 5552 & 321 & 5540.5 & 5526.0 & 0.01951 & 0.04 \\
\hline
\end{tabular}

The first four columns of this table identify the problem by its name, $m, n$, and $z^{\mathrm{LP}}$, the known optimal value of the linear programming relaxation. The 
remaining columns are related to the volume algorithm [1], namely, the number of iterations up to finding a primal vector where each constraint is violated by at most 0.02 or the difference between the dual lower bound and the primal value is less than $1 \%$. We also present the time required by the volume algorithm (in seconds).

From the (dual) lower bound derived from volume algorithm we obtain an integral lower bound $z_{a}$ on the value of $z_{*}$. The initial $x_{a}$ was set to all-zeros because often the primal solution obtained through the volume algorithm would not satisfy $c x \leq z_{a}$. Note that, since $c>0, x_{a} \in P^{\prime}\left(z_{a}\right)$. In our experiments we have chosen to set $\Delta=1$.

We implemented the first-order feasible directions minimization algorithm, presented in Sect. 3, to solve the nonlinear problem (3). In Step 2 of the Solve_ subproblem algorithm we used the software CpLex (version 7.1) to get an optimal solution for the linear problem.

Table 2 is divided into four parts. The first part identifies the set-partitioning problem. Next, we have the initial value for $z_{a}$ (input to Main), that is the lower bound found by volume algorithm.

Table 2. Results of solving (3) with first-order and second-order implementations

\begin{tabular}{lrrrrrrrrr}
\hline & & \multicolumn{4}{c}{ First-order } & \multicolumn{3}{c}{ Second-order } \\
\cline { 3 - 10 } Name & Initial & Final & \multicolumn{2}{c}{ Max. } & Out. & Time & \multicolumn{2}{c}{ Max. } & Out. Time \\
& \multicolumn{1}{c}{$z_{a}$} & $\varepsilon$ & Viol. & It. & (sec.) & Viol. & It. & (sec.) \\
\hline sppnw08 & 35894 & $6.10 \mathrm{E}-05$ & $4.20 \mathrm{E}-07$ & 1 & 469.86 & $1.28 \mathrm{E}-14$ & 1 & 21.48 \\
sppnw10 & 68147 & 0.01563 & 0.00269 & $4^{*}$ & 26404.26 & $9.15 \mathrm{E}-10$ & 6 & 3546.41 \\
sppnw12 & 14102 & 0.00781 & 0.00121 & $3^{*}$ & 17301.78 & $7.41 \mathrm{E}-14$ & 4 & 134.80 \\
sppnw15 & 67714 & 0.03125 & 0.00521 & $1^{*}$ & 4522.86 & $5.79 \mathrm{E}-09$ & 5 & 124.87 \\
sppnw20 & 16604 & 0.03125 & 0.00819 & $1^{*}$ & 5653.53 & $9.88 \mathrm{E}-09$ & 4 & 275.65 \\
sppnw21 & 7371 & 0.03125 & 0.00573 & $1^{*}$ & 4752.56 & $8.96 \mathrm{E}-10$ & 4 & 110.24 \\
sppnw22 & 6917 & 0.01563 & 0.00470 & $3^{*}$ & 7919.76 & $2.68 \mathrm{E}-10$ & 5 & 230.26 \\
sppnw23 & 12301 & 0.01563 & 0.00385 & $2^{*}$ & 9034.06 & $2.74 \mathrm{E}-11$ & 5 & 15173.3 \\
sppnw24 & 5828 & 0.01563 & 0.00296 & $3^{*}$ & 44584.35 & $1.38 \mathrm{E}-09$ & 4 & 2107.83 \\
sppnw25 & 5830 & 0.01563 & 0.00280 & $6^{*}$ & 59902.49 & $1.14 \mathrm{E}-09$ & 5 & 1718.74 \\
sppnw26 & 6733 & 0.01563 & 0.00308 & $3^{*}$ & 16722.60 & $1.91 \mathrm{E}-08$ & 4 & 275.63 \\
sppnw27 & 9871 & 0.00781 & 0.00175 & $3^{*}$ & 23535.21 & $2.18 \mathrm{E}-14$ & 5 & 1588.36 \\
sppnw28 & 8161 & 0.00781 & 0.00104 & $4^{*}$ & 23150.30 & $8.65 \mathrm{E}-15$ & 4 & 1147.54 \\
sppnw32 & 14560 & 0.01563 & 0.00296 & $3^{*}$ & 3059.28 & $8.72 \mathrm{E}-10$ & 4 & 12.51 \\
sppnw35 & 7194 & 0.01563 & 0.00393 & $2^{*}$ & 19234.21 & $1.92 \mathrm{E}-09$ & 5 & 5420.31 \\
sppnw36 & 7260 & 0.01563 & 0.00302 & $1^{*}$ & 32498.39 & $4.23 \mathrm{E}-13$ & 1 & 3049.8 \\
sppnw38 & 5541 & 0.01563 & 0.00457 & $2^{*}$ & 26496.43 & $1.43 \mathrm{E}-10$ & 4 & 1677.83 \\
\hline
\end{tabular}

In the third part, we present the results of the first-order implementation. For all these problems the initial value for $\varepsilon$ is 1 , because at the beginning of the algorithm the primal vector $x_{a}$ is a vector of zeros and $\varepsilon=\left\|\mathbb{1}-A x_{a}\right\|_{\infty}=1$. We exhibit the final value for $\varepsilon$, the maximal violation obtained for the constraints, the number of outer iterations, which corresponds to the number of bisection calls, and the time (in seconds) required. For all problems, except sppnw08, no optimal $\bar{x}$ was found (flag=CP4), as the maximum number of iterations 
(set to 20000) was reached, as pointed out with the character $*$ in the table. Nevertheless, the maximal constraint violation obtained is reduced at least $60 \%$ (compare with Table 1).

Then we analyze the strategy of solving the nonlinear subproblem (3) using solver LOQO 6.0. LOQO is an implementation of a primal-dual interior point method for solving nonlinear constrained optimization problems. The fourth part of Table 2 summarizes the results. We remark that, for all the problems, the algorithm halted because the stopping criterion in the Main algorithm $\left(\varepsilon<10^{-4}\right)$ was achieved. The maximal violation of the constraints is now much smaller than the one obtained with the volume algorithm. A different $\varepsilon$ reduction scheme $(\varepsilon:=\varepsilon / 10)$ in the Step 2 of the Main algorithm was tried and, in general, the number of outer iterations decreased by one or two units and the time spent was smaller in $71 \%$ of the problems.

\section{Conclusions}

In this paper we propose an algorithm for finding an $\varepsilon$-feasible solution relatively to the constraints of a linear program. The first-order version of the algorithm attempts to minimize a linear approximation of an exponential penalty function using feasible directions and a constant stepsize. The second-order implementation uses the software LOQO to minimize the exponential function.

Our preliminary computational experiments show that the first-order method does not perform as expected when solving set-partitioning problems. In particular, the algorithm converges very slowly and does not reach an $\varepsilon$-approximate solution, for $\varepsilon<10^{-4}$, for all tested problems but for sppnw08.

We may also conclude that the second-order implementation of the algorithm works quite well, specially for problems that are not large-scale, finding an $\varepsilon$ feasible solution in (1) satisfying $c x<z$, for a small and adequate $\varepsilon$ value. Future developments will focus on improving convergence of the first-order algorithm.

\section{References}

1. Barahona, F., Anbil, R.: The volume algorithm: Producing primal solutions with a subgradient method. Math. Prog. 87 (2000) 385-399

2. Bienstock, D.: Potential Function Methods for approximately solving linear programming problems: theory and practice. Kluwer Academic Publishers (2002)

3. Grigoriadis, M.D., Khachiyan, L.G.:Fast approximation schemes for convex programs with many blocks and coupling constraints. SIAM J. Optim. 41994 86-107

4. Karger, D., Plotkin, S.: Adding multiple cost constraints to combinatorial optimization problems, with applications to multicommodity flows. Proceedings of the 27th Annual ACM Symposium on Theory of Computing (1995) 18 - 25

5. Plotkin, S., Shmoys, D.B., Tardos, E.: Fast approximation algorithms for fractional packing and covering problems. Math. Oper. Res. 20 (1995) 495-504

6. Rocha, A.M.: Fast and stable algorithms based on the Lagrangian relaxation. PhD Thesis (in portuguese), Universidade do Minho, Portugal (2004) (forthcoming) 